\title{
EFECTIVIDAD DE LA CREMA DE PROPÓLEOS EN EL TRATAMIENTO DE LAS QUEILITIS POR CONTACTO
}

Effectiveness of propolis cream in the treatment of contact cheilitis

Fecha de Recepción: 21 de julio 2021
Eficácia do creme de própolis no tratamento da queilite de contato

Aceptado para su publicación: 30 de septiembre 2021
Víctor Ricardo Fernández ${ }^{1,}$ Elena Soledad Ortiz Barreto ${ }^{1, b}$ Roque Oscar Rosende ${ }^{1, a}$

María Susana Briend ${ }^{1, c}$

1. Módulos de Patología y Diagnostico I, II y III. Facultad de Odontología. UNNE. Argentina.

a. Doctor de la Universidad Nacional del Nordeste en Odontología (UNNE)

b. Especialista en Docencia y Gestión Universitaria (UNNE)

c. Master en Oncología Molecular (European School of Oncology)

Correspondencia:

Fernández, Víctor Ricardo Facultad de Odontología, Campus Deodoro Roca Av. Libertad 5450, W3400 Corrientes

+5403794457992

Correo electrónico: fernandezvr@hotmail.com vrfernandez@odn.unne.edu.ar

Conflicto de intereses: los autores declaran no tener conflictos de interés.

Fuente de financiamiento: Secretaría General de Ciencia y Técnica. Universidad Nacional del Nordeste.

\section{Resumen}

Las queilitis por contacto son procesos patológicos de etiología multifactorial de origen externo que generan lesiones en el bermellón de los labios, en algunos casos se acompaña por una dermatitis contigua. Este tipo de queilitis recibe esta denominación debido al mecanismo de acción de la noxa productora, la cual actúa por contacto en la superficie labial produciendo una reacción antígeno anticuerpo. Los agentes sensibilizantes y desencadenantes del proceso inflamatorio son de origen físico y químico en sus diversas formas. Los físicos son los rayos actínicos y ultravioletas. Los químicos son más numerosos, como los cosméticos, alimentos, pastas y cepillos dentales. La incidencia es alta en el sexo femenino y en el labio inferior por su mayor superficie. El objetivo de este estudio fue realizar una evaluación comparativa de la crema de propóleos en el tratamiento de las queilitis por contacto y de la posible reacción adversa medicamentosa. Se llevó a cabo en pacientes de ambos sexos, a partir de los 15 años con diagnóstico clínico de queilitis por contacto, los que fueron asignados en forma aleatorizada simple en dos grupos. El primer grupo de control, recibió tratamiento tradicional. El segundo grupo experimental recibió la formulación objeto de estudio, la crema de propóleos. Ambas medicaciones redujeron de manera significativa la intensidad de la sintomatología y los signos sin efectos adversos en pacientes con queilitis por contacto. La formulación magistral de crema de propóleos objeto del presente estudio, ha demostrado ser efectiva en el tratamiento de las queilitis por contacto.

Palabras clave: queilitis, labio, inflamación, terapéutica (fuente: DeCS BIREME). 


\section{INVESTIGACIÓN}

Efectividad de la crema de propóleos en el tratamiento de las queilitis por contacto
ISSN 1668-7280

ISSN-E 2683-7986

\begin{abstract}
Contact cheilitis are pathological processes of multifactorial etiology of external origin that generate lesions on the vermilion of the lips. In some cases, it also involves the nearby skin generating dermatitis. This type of cheilitis is so called due to the mechanism of action of the noxa, which acts by contact on the labial surface producing an antigen-antibody reaction. The sensitizing and triggering agents of the inflammatory process are of physical and chemical origin in their various forms. The physical ones are actinic and ultraviolet rays. The chemical ones are more numerous, such as cosmetics, food, kinds of toothpaste and toothbrushes. The incidence is high in females and on the lower lip due to its larger surface. This study aimed to perform a comparative evaluation of propolis cream in the treatment of contact cheilitis and possible adverse drug reaction. It was performed both in male and female patients aged 15 years of age and older with a clinical diagnosis of contact cheilitis, who were randomized into two groups. The control group received traditional treatment. The experimental group received the formulation under study, propolis cream. Both medications significantly reduced the intensity of symptoms and signs without adverse effects in patients with contact cheilitis. The propolis cream formulation studied in this research is effective in the treatment of contact cheilitis.
\end{abstract}

Key words: cheilitis, lip, inflammation, therapeutics (source: MeSH NLM).

\section{Resumo}

A queilite de contato é um processo patológico de etiologia multifatorial de origem externa que gera lesões no vermelhão dos lábios, podendo em alguns casos comprometer também a pele próxima, gerando dermatites. Esse tipo de queilite recebe esse nome devido ao mecanismo de ação de noxa, que atua por contato na superfície labial produzindo uma reação antígeno-anticorpo. Os agentes sensibilizantes e desencadeadores do processo inflamatório são de origem física e química em suas diversas formas. Os físicos são os raios actínicos e ultravioleta. Os produtos químicos são mais numerosos, como cosméticos, alimentos, pastas de dente e escovas de dente. A incidência é alta no sexo feminino e no lábio inferior devido à sua superfície maior. O objetivo deste estudo foi realizar uma avaliação comparativa do creme de própolis no tratamento da queilite de contato e possível reação adversa medicamentosa. Foi realizado em pacientes de ambos os sexos, a partir dos 15 anos com diagnóstico clínico de queilite de contato, os quais foram alocados de forma randomizada simples em dois grupos. O primeiro grupo de controle recebeu tratamento tradicional. O segundo grupo experimental recebeu a formulação em estudo, o creme de própolis. Ambos os medicamentos reduziram significativamente a intensidade dos sintomas e sinais sem efeitos adversos em pacientes com queilite de contato. A formulação magistral do creme de própolis, objeto deste estudo, tem se mostrado eficaz no tratamento da queilite de contato.

Palavras-chave: queilite; lábio, inflamação, terapêutica (fonte: DeCS BIREME).

\section{Introducción}

Las queilitis son procesos inflamatorios inespecíficos que asientan en el borde libre labial y comisuras. Presentan un cuadro histológico inespecífico y su etiología es multifactorial ${ }^{1-3}$. La mayor incidencia se registra en el labio inferior por poseer una mayor superficie que favorece la acción de los irritantes. Según la afectación de las glándulas salivales accesorias se pueden distinguir en glandulares y no glandulares $^{4-7}$.

Las queilitis por contacto son reacciones inflamatorias localizadas en la semimucosa labial y en la porción cutánea contigua, caracterizada por descamaciones, erosiones y fisuras ${ }^{8-10}$. Son unas de las formas más frecuentes de queilitis del tipo reversible y su evolución permite una restitución a la normalidad anatómica y funcional. Generalmente son de corta duración y con una muy alta incidencia ${ }^{11,12}$.

La lesión se localiza esencialmente en el bermellón de los labios, en respuesta a la acción de irritantes externos de acción local que actúan por un mecanismo de hipersensibilidad ${ }^{13,14}$. Estas noxas pueden ser de origen físico o químico e inician un proceso inflamatorio que se presenta como un eritema acompañado de aumento de tamaño y eversión labial en algunos casos, que posteriormente se fisura y se extiende a la piel ${ }^{15,16}$. Las noxas primarias inducen a la formación de sustancias histaminoides que aumentan la permeabilidad vascular en la piel y muco- 


\section{REFO}

VOL. XIV | N² 2| AÑO 2021

ISSN 1668-7280

ISSN-E 2683-7986 sa que a su vez son las causantes de las lesiones. Los agentes lesivos o contactantes provocan eczemas o lesiones eczematoides ${ }^{17,18}$.

Frecuentemente las lesiones son superficiales, provocando aumento en el volumen del o de los labios, a veces con eversión de los mismos. El cuadro se inicia de forma aguda con una duración de 15 días siempre que se identifique y anule el agente causal. En el estado agudo los labios aparecen edematosos, rojos, calientes y dolorosos. Se erosionan y se cubren posteriormente de costras serohemáticas que al ser arrancadas dejan ver fisuras verticales. En la mayoría de los casos las lesiones se producen por lo general en ambos labios y se extienden a la piel ${ }^{19,20}$.

Epidemiológicamente es una patología cada vez más frecuente debido al incremento de exposición a la luz solar, uso de cosméticos, alimentos, medicamentos en especial tópicos que contienen galatos, dentífricos, cepillos dentales, metales, prótesis dentales, plantas, otras sustancias exógenas (químicas o físicas) que determinan una incidencia mayor en personas jóvenes con localización en el labio inferior y preferentemente en el sexo femenino ${ }^{21-26}$.

Ante la acción repetida de las noxas, esencialmente las de origen físico y los factores coadyuvantes o predisponentes favorecen la cronicidad del proceso con tendencia a la malignidad. La histopatología muestra degeneración basófila del componente colágeno, daño en la vascularización y el epitelio de revestimiento ${ }^{27-30}$. El epitelio sufre atrofia volviéndose más vulnerable a la acción de carcinógenos. El diagnóstico precoz de las queilitis por contacto permite la identificar la noxa y suprimirla, aunque en ocasiones es muy difícil identificarla ${ }^{31-35}$.

El tratamiento de las queilitis de contacto consiste en la aplicación tópica de sustancias como los derivados del propóleos. Se han reportado en estomatología estudios sobre efectos beneficiosos de los propóleos en el tratamiento de lesiones gingivales, ulceras y aftas bucales, así también en la terapéutica de la alveolitis, de las lesiones periodontales y como regenerador de los epitelios en las queilitis por contacto $^{36,37}$.
En base a las propiedades citadas, el propósito del trabajo consistió en la evaluación comparativa de la efectividad de la crema de propóleos con la medicación tradicional en el tratamiento de las queilitis por contacto.

\section{Materiales y Métodos}

El presente es un estudio comparativo, controlado, abierto sobre la utilización de la crema de propóleos en pacientes con diagnóstico de queilitis por contacto. Se llevó a cabo mediante un protocolo de investigación aprobado por el Comité de Bioética de la Facultad de Odontología de la Universidad Nacional del Nordeste.

El trabajo se realizó en pacientes de ambos sexos, comprendidos entre los 15 y 50 años de edad, que concurrieron en forma espontánea a la clínica asistencial de los Módulos de Patología y Diagnóstico I, II y III, del Servicio de Estomatología y del Hospital Odontológico de la FOUNNE, en demanda de atención. Previo consentimiento por escrito, se distribuyeron en forma aleatoria simple para conformar dos grupos. El primer grupo se denominó grupo experimental, recibió la formulación objeto de estudio, la crema de propóleos (Cp) cuya composición es extracto espeso de propóleos $5 \mathrm{~g}$, vaselina $80 \mathrm{~g} \mathrm{y}$ lanolina $15 \mathrm{~g}$. En el segundo grupo identificado como grupo de control se aplicó con el tratamiento tradicional $(\mathrm{Mt})$ en base a betametasona valerato al 0,1\%. El tratamiento se suministró con una posología de cuatro aplicaciones diarias. Ambos grupos estuvieron conformados lo más equitativos posible según el sexo. Fueron excluidos los pacientes que padecían enfermedades de alto riesgo médico, los que consumían sustancias adictivas o con inestabilidad domiciliaria, con antecedentes de alergia o paciente con antecedentes de atopía o cualquier otra situación médica que a juicio del investigador fue considerada inapropiada su inclusión. Se consideró como criterio de salida a los pacientes que interrumpieran el tratamiento o dejaran de asistir a las evaluaciones clínicas.

Se realizaron exámenes estomatológicos a 70 pacientes, a 35 se los incluyó en el grupo experimental tratados con la formulación objeto de estudio, 
REFO

VOL. XIV | No 2| AÑO 2021

ISSN 1668-7280

ISSN-E 2683-7986 crema de propóleos al $5 \%$ y a 35 se los incluyó en el grupo control tratados con la medicación tradicional.

Se procedió a la atención de los pacientes en la clínica, se les informó sobre el proyecto de investigación. Los pacientes que accedieron a formar parte de la investigación, consintieron por escrito y posteriormente fueron registrados en planillas diseñadas para el control y la evolución.

Los controles y las evaluaciones del tratamiento se realizaron en ambos grupos, en varios tiempos operatorios, identificados como:

- Tiempo 0 (TO), correspondió a la eliminación de la causa, a la toma de muestras para el primer estudio citológico y a la administración de las fórmulas magistrales según corresponda a cada grupo.

- $\quad$ Tiempo 1 (T1), correspondió al primer control a realizarse a los 3 días y a la toma de muestras para el segundo estudio citológico.

Tiempo 2 (T2), correspondió al segundo control a realizarse a los 7 días, a la toma de muestras para el tercer estudio citológico y a la suspensión del tratamiento.

En cada control se consignó el estado de la lesión de acuerdo a las resultantes de la valoración de la sintomatología mediante una escala de Downie modificada 38 y de los signos, mediante una escala milimetrada de la superficie afectada, identificadas como:

Estado 1 (E1) o de leve mejoría (reducción en un $25 \%$ de los síntomas y signos registrados en el tiempo 0).

- $\quad$ Estado 2 (E2) o de franca mejoría (reducción en un $50 \%$ de los síntomas y signos registrados en el tiempo 0).

- $\quad$ Estado 3 (E3) o de cura completa (remisión en un $100 \%$ de los signos y síntomas).

- $\quad$ Estado 4 (E4) o de persistencia de la lesión.
Se tomaron muestras para citología exfoliativa (Cex) por frotis convencionales. Se fijó en solución alcohólica, se dejó secar al aire, durante cinco minutos y se colocó dentro de un sobre de papel, identificado con el número de caso y se adjuntó el protocolo de estudio citológico diseñado para tal efecto. Los extendidos fueron procesados en el Servicio de Anatomía Patológica de la FOUNNE, coloreados con la técnica de Papanicolaou (Hematoxilina, EA36, Orange). Finalmente, observados y evaluados con microscopio óptico a 100X y 400X para determinar la calidad y representatividad de las muestras para arribar a conclusiones valederas.

Se registraron los datos observados en Protocolos Sistematizados de Citología utilizados por el Servicio. Se utilizó un mismo observador para evitar interpretaciones erróneas. En base a esta identificación se pudo abordar a los siguientes diagnósticos citológicos especificando: Si hay inflamación (Dc1) y No hay inflamación (Dc2).

Para comprender la estructura de los datos se utilizó estadística descriptiva. Para evaluar los grupos (evaluación clínica y citológica) se utilizó la Prueba de Chi Cuadrado de Pearson.

\section{Resultados}

Los controles de la evolución realizados en los 35 pacientes del Grupo Experimental tratados con $\mathrm{Cp}$ permitieron registrar que, en el T0, se realizó la citología exfoliativa de las lesiones con un resultado de Dc1 en 35 pacientes (100\%). En el T1, el 80\% de pacientes (28) lograron un E1 y el $22 \%$ de pacientes (7) alcanzaron un E2. En el T2, el 91,4\% de pacientes (32) lograron un E3 y el 8,5\% de pacientes (3) un E2.

Estudio citológico del grupo experimental realizada en el T0 y en el T1, registró Dc1 en 100\% de los pacientes (35). Después de la administración de las formulaciones (T2 - Cex 3) dio como resultado que en los pacientes del grupo experimental en el $94,2 \%$ de casos (33) logró una valoración citológica de Dc2 y el 5,7\% de casos (2) obtuvo una valoración citológica de Dc1.

Los controles de la evolución realizados en los 35 pacientes del Grupo Control tratados con Mt permitieron registrar que: En el T0, se realizó la citología 
INVESTIGACIÓN
Revista de la Facultad de Odontología

Fernández | Ortiz Barreto | Rosende | Briend

http://dx.doi.org/10.30972/rfo.1425764

\section{REFO}

VOL. XIV | N² 2| AÑO 2021

ISSN 1668-7280

ISSN-E 2683-7986 exfoliativa de las lesiones con una valoración citológica de Dc1 en el 100\% de pacientes. En el T1, el 60\% de los pacientes (21) alcanzaron un estado de leve mejoría, el 28,5\% de los pacientes (10) alcanzaron un estado de franca mejoría y el $11,5 \%$ de los pacientes (4) hubo persistencia de la lesión. En el T2, el 82,8\% de los pacientes (29) lograron un E3 y el 17,2\% de los pacientes (6) un E2. El estudio citológico en el grupo control realizado en el T0 y en el T1 registró Dc1 en el $100 \%$ los casos (35 pacientes) al igual que el grupo experimental.

La administración de las formulaciones (T2 - Cex 3) dio como resultado que en el $88,5 \%$ de los casos del grupo control (31) logró una valoración citológica de Dc2 y el $11,5 \%$ de casos (4) obtuvo una valoración citológica de Dc1.

La comparación de las evoluciones de ambos grupos en el primer control a los 3 días demostró que la medicación experimental logró mejores resultados ya que el $100 \%$ de los pacientes alcanzaron un E2 y E1. En comparación con el grupo control que el $60 \%$ alcanzaron un E1, el 28,5\% alcanzaron un E2 y el 11,5 \% con E4 (Tabla I). La comparación de las evoluciones de ambos grupos en el segundo control a los 7 días permitió obtener el resultado de que en ambos grupos se lograron E3 y E2 en el 100\% de los casos. La comparación de los estadios evolutivos de ambos grupos reveló una supremacía del grupo experimental con un $91,5 \%$ de $\mathrm{E} 3$ y $8,5 \%$ de $\mathrm{E} 1$, sobre el grupo control con un $82,8 \%$ de $E 3$ y el $17,2 \%$ de E2 (Tabla II).

Respecto de la evaluación citológica de los casos estudiados arroja el resultado comparativo que en el T1 y T2 se registró el 100\% de Dc1 como valoración citológica. Se registró una supremacía en el T2 en el grupo experimental, las valoraciones citológicas correspondientes al $94,2 \%$ como Dc2 y el $5,7 \%$ como Dc1. En el grupo control las valoraciones citológicas registradas correspondieron Dc2 en el $88,5 \%$ y del 11,5\% como Dc1 respectivamente (Tabla III).

El análisis estadístico de las evaluaciones clínicas arrojó un p-valor de 0,0620 en el T1 y un p-valor de 0,2841 en el T2, estadísticamente no significativas. En las evaluaciones citológicas el análisis estadísti-

Tabla I. Resultados de la evaluación clínica de los grupos a los tres días.

\begin{tabular}{c|l|l|l|l|l|l} 
Tiempo & Grupo & E1 & E2 & E3 & E4 & Total \\
T1 & Control & 21 & 10 & 35 \\
T1 & Experimental & 28 & 7 & 0 & 0 \\
T1 & Total & 49 & 17 & 0 & 45 \\
\hline
\end{tabular}

Tabla II. Resultados de la evaluación clínica de los grupos a los siete días.

\begin{tabular}{c|l|l|l|l|l|l} 
Tiempo & Grupo & E1 & E2 & E3 & E4 & Total \\
T2 & Control & 0 & 6 & 29 & 35 \\
T2 & Experimental & 0 & 3 & 32 & 0 \\
T2 & Total & 0 & 9 & 61 & 0 \\
\hline
\end{tabular}

Tabla III. Resultados de la evaluación citológica de los grupos a los siete días.

\begin{tabular}{c|l|l|l|l} 
Tiempo & Grupo & Dc1 & Dc2 & \\
T2 & Control & 4 & 31 & 35 \\
T2 & Experimental & 2 & 33 & 35 \\
T2 & Total & 6 & 64 & 70 \\
\hline
\end{tabular}




\section{INVESTIGACIÓN}

co aplicado arrojó un p-valor de 0,9999 en el T1 y un $p$ - valor de 0,3932 en el T2, estadísticamente no significativas.

Los resultados encontrados como E3 de las lesiones en el T2, indica que en el grupo experimental alcanzo al 91,4\% de pacientes tratados, en relación al $82,8 \%$ logrado en el grupo control, dan cuenta de una superioridad clínica de la formulación objeto de estudio en comparación con la medicación tradicional. Ambas medicaciones reducen de manera significativa la intensidad de la sintomatología y los signos sin efectos adversos en pacientes con queilitis por contacto.

\section{Discusión}

Las queilitis por contacto relevadas de las historias clínicas de los pacientes incluidos en ambos grupos, responden a noxas similares a las citadas por Zhou et al. ${ }^{28}$, van Amerongen et al. ${ }^{29}$, de Groot ${ }^{30}$ y Horst et al. ${ }^{31}$, siendo las responsables de las manifestaciones clínicas caracterizadas por presentar signos como aumento de tamaño, eversión y aspecto eritematoso. En la etapa aguda, los pacientes refirieron calor y dolor, análogos síntomas fueron citados por Ceccotti et al. . . Cuando el cuadro anatomopatológico evoluciona hacia la cronicidad pueden presentarse en la semimucosa costras serohemáticas producto de la deshidratación del exudado que se inicia a partir del arrancamiento de las escamas, pudiéndose extenderse a la piel. Las características clínicas observadas en los casos analizados guardan similitud con los factores productores registrados en la bibliografía consultada ${ }^{11,12}$.

La aplicación de presentaciones farmacológicas en base al propóleos, demostró su efectividad en la disminución de los signos y síntomas, en la remisión del cuadro clínico y consecuentemente la recuperación de la salud y funcionalidad de los labios. Los resultados alcanzados son análogos a los obtenidos por Macias ${ }^{39}$, quien reportó una acción directa de propóleos sobre el proceso de reepitelización. Por su parte, Noriega Salmon ${ }^{35}$, afirma que un extracto acuoso de propóleos actúa como un buen anestésico local y provee de una acción infiltradora similar a la procaína, condición sintomática registrada en los controles efectuados en nuestro trabajo.
Los datos de toxicidad del propóleo son limitados. Sin embargo, se han informado varios informes de casos de reacciones alérgicas. Pueden manifestarse como queilitis de contacto, estomatitis de contacto, eczema perioral, edema labial, dolor oral, descamación de los labios y disnea ${ }^{40}$. En nuestro estudio, no se registraron reacciones adversas a diferencia del reporte del caso publicado por Budimir et al. ${ }^{41}$, quienes observaron luego de una automedicación de propóleos en aerosol, edema labial y eritema de la piel perioral acompañado de ardor en los labios.

\section{Conclusiones}

La formulación magistral de crema de propóleos objeto del presente estudio comparativo, ha demostrado ser efectiva en el tratamiento de las queilitis por contacto. La crema de propóleos permitió la remisión del cuadro clínico y de la reducción de los signos y síntomas. La formulación es de aplicación tópica y de fácil manipulación, carente de efectos adversos. Se recomienda el uso del extracto espeso de propóleos al $5 \%$, en el tratamiento de las queilitis por contacto.

\section{Referencias Bibliográficas}

1. Lugović-Mihić L, Blagec T, Japundžić I, Skroza N, Delaš Adžajić $M$, Mravak-Stipetić M. Diagnostic management of cheilitis: an approach based on a recent proposal for cheilitis classification. Acta Dermatovenerol Alp Pannonica Adriat, 2020;29(2): 67-72. doi: 10.15570/actaapa.2020.16

2. Romeo U, Rocchetti F, Montori A. Criticisms and Controversies in the Diagnosis of Cheilitis. Multidisciplinary Digital Publishing Institute Proceedings. 2019.35 (1), 8. doi: 10.3390/proceedings2019035008

3. Ceccotti E, Sforza R. El Diagnóstico en Clínica Estomatológica. Buenos Aires: Editorial Panamericana; 2007.

4. Lugović-Mihić L, Pilipović K, Crnarić I, Šitum M, Duvan ĉić T. Differential Diagnosis of Cheilitis - How to Classify Cheilitis? Acta Clin Croat. 2018 Jun;57(2):342-351. doi: 10.20471/acc.2018.57.02.16

5. Eberting C, Blickenstaff N, Goldenberg A. Pathophysiologic Treatment Approach to Irritant Contact Dermatitis. Curr Treat Options Allergy. 2014;1(4)317-328. https:// doi.org/10.1007/s40521-014-0030-0

6. Budimir J, Mravak-Stipetić M, Bulat V, Ferĉek I, Japundžić I, Lugović-Mihić L. Allergic reactions in oral and perioral diseases-what do allergy skin test results show? Oral Surg Oral Med Oral Pathol Oral Radiol. 2019;127(1):40- 
Revista de la Facultad de Odontología

Fernández | Ortiz Barreto | Rosende | Briend

http://dx.doi.org/10.30972/rfo.1425764

\section{REFO}

VOL. XIV | N² 2| AÑO 2021

ISSN 1668-7280

ISSN-E 2683-7986
48. https://doi.org/10.1016/j.00oo.2018.08.001

7. Bakula A, Lugović-Mihić L, Situm M, Turcin J, Sinković A. Contact allergy in the mouth: diversity of clinical presentations and diagnosis of common allergens relevant to dental practice. Acta Clin Croat. 2011;50(4):553-61. Disponible en: https://pubmed.ncbi.nlm.nih.gov/22649886/

8. Schwarz I, Bokanovic D, Aberer W. Mucosal diseases from an allergological perspective.Der Hautarzt; Zeitschrift fur Dermatologie, Venerologie, und Verwandte Gebiete. 2016; 67 (10): 780-785. doi: 10.1007 / s00105016-3866-3

9. Gagari E. Cheilitis and Oral Disease. In: European Handbook of Dermatological Treatments. Katsambas AD, Lotti T, Dessinioti C, D’Erme AM (Eds.). Berlin: Springer-Verlag Berlin Heidelberg; 2015.

10. Collet E, Jeudy G, Dalac S. Cheilitis, perioral dermatitis and contact allergy. Eur J Dermatol. 2013; 23(3):303-7. doi: 10.1684 / ejd.2013.1932

11. Oakley A. Cheilitis. | DermNet [Internet]. 2010 [cited 2020 Apr 3]. [about 1 p.]. Disponible en: https://www. dermnetnz.org/topics/cheilitis/.

12. O'Gorman S, Torgerson R. Contact allergy in cheilitis. Int J Dermatol. 2016; 55(7):386-91. doi: 10.1111/ ijd.13044

13. Chalkoo A, Makroo N, Peerzada G. Exfoliative cheilitis. Indian JDentAdv.2016;8:3-9.doi:10.5866/2016.8.10056 14. Almazrooa S, Woo S, Mawardi H, Treister N. Characterization and management of exfoliative cheilitis: a single-center experience. Oral Surg Oral Med Oral Pathol Oral Radiol. 2013;116(6):e485-9. doi: 10.1016/j. oooo.2013.08.016. PMID: 24237731

15. Rosińska-Więckowicz A, Misterska M, Bartoszak L, Żaba R. Case report. Cheilitis - case study and literature review. Post Dermatol Alergol. 2011;28(3):231-239. Disponible en: https://www.termedia.pl/Case-report-Cheilitis-case-study-and-literature-review,7,16943,1,1.html
16. Van Baelen A, Kerre S, Goossens A. Allergic contact cheilitis and hand dermatitis caused by a toothpaste. Contact Dermatitis. 2016;74(3)187-189. doi: 10.1111/ cod. 12505

17. Bansal S, Shaikh S, Desai R, Ahmad I, Puri P, Prasad $P$, et al. Spectrum of Lip Lesions in a Tertiary Care Hospital: An Epidemiological Study of 3009 Indian Patients. Indian Dermatol Online J. 2017;8(2):115-119. doi: 10.4103/2229-5178.202280

18. Lugović-Mihić L, Ilić I, Budimir J, Pondeljak N, Mravak Stipetić M. Common allergies and allergens in oral and perioral diseases. Acta Clin Croat. 2020;59(2):318-328. doi: 10.20471/acc.2020.59.02.16

19. Hitz Lindenmüller I, Itin P, Fistarol S. Dermatology of the lips: inflammatory diseases. Quintessence International (Berlin, Germany: 1985). 2014;45(10):875-883. doi: 10.3290/j.qi.a32638

20. Greenberg S, Schlosser B, Mirowski G. Diseases of the lips. Clin Dermatol. 2017;35(5):e1-e14. doi: 10.1016/j. clindermatol.2017.11.003

21. Li Y, Li L. Contact Dermatitis: Classifications and Management. Clin Rev Allergy Immunol. 2021. doi: 10.1007/ s12016-021-08875-0

22. Minciullo P, Paolino G, Vacca M, Gangemi S, Nettis E. Unmet diagnostic needs in contact oral mucosal allergies. Clin Mol Allergy. 2016;14(10)1-8. doi: 10.1186/s12948016-0047-y

23. Mizutani $\mathrm{H}$. Causes of allergic contact cheilitis in Melbourne. Australasian Journal of Dermatology. 2019;60(Suppl. 1):65. Australasian College of Dermatologists Abstracts Presented at the 52nd Annual Scientific Meeting 2019. Disponible en: https://dermcollabstracts. com/abstract/causes-of-allergic-contact-cheilitis-in-melbourne/

24. Gonzaga A, de O. Bezerra H, Cavalcante I, Santana T, de Oliveira P, de Medeiros P, et al. An update about chei- 


\section{INVESTIGACIÓN}

Efectividad de la crema de propóleos en el tratamiento de las queilitis por contacto
ISSN 1668-7280

ISSN-E 2683-7986 litis. J Oral Maxillofac Surg Med Pathol. 2021;33(5):555560. doi: 10.1016/j.ajoms.2021.02.001

25. Samimi M. Chéilites: orientation diagnostique et traitement [Cheilitis: Diagnosis and treatment]. Presse Med. 2016;45(2):240-50. doi: 10.1016/j.Ipm.2015.09.024

26. Hitz Lindenmüller I, Itin P, Fistarol S. Dermatology of the lips: inflammatory diseases. Quintessence Int. 2014;45(10):875-83. doi: 10.3290/j.qi.a32638

27. Sánchez-Herrero A, Mateos-Mayo A, Rodríguez-Lomba E, Molina-López I, Campos-Domínguez M, Suárez Fernández R. Allergic contact cheilitis in an adolescent to Ricinus communis seed oil (castor oil) in a lip balm. Contact Dermatitis. 2018;79(3):176-178. doi: 10.1111/cod.13016 28. Zhou L, Pratt M. Allergic Contact Cheilitis From a Variety of Lip Balm Ingredients. J Cutan Med Surg. 2018;22(3):333-335. doi: 10.1177/1203475417738969 29. van Amerongen C, de Groot A, Volkering R, Schuttelaar M. Cheilitis caused by contactallergy to toothpaste containing stannous (tin) - two cases. Contact Dermatitis. 2020;83(2)126-129. doi: 10.1111/cod.13532

30. de Groot A. Contact allergy to (ingredients of) toothpastes. Dermatitis. 2017;28(2):95-114. doi: 10.1097/ DER.0000000000000255

31. Horst N, Leysen J, Mellaerts T, Lambert J, Aerts O. Allergic contact cheilitis from anethole-containing toothpastes: a practical solution. J Eur Acad Dermatol Venereol. 2017;31(8):e374-e375. doi: 10.1111/jdv.14174

32. Rozas-Muñoz E, Lepoittevin J, Pujol R, Giménez-Arnau A. Allergic contact dermatitis to plants: understanding the chemistry will help our diagnostic approach. Actas Dermosifiliogr. 2012;103(6):456-77. doi: 10.1016/j. ad.2011.07.017

33. Barakian Y, Vahedi M, Sadr P. Exfoliative Cheilitis: A Case Report, Avicenna J Dent Res. 2015 ;7(2):e24943. doi: 10.17795/ajdr-24943.

34. Holcomb Z, Van Noord M, Atwater A. Gallate Con- tact Dermatitis: Product Update and Systematic Review. Dermatitis. 2017;28(2):115-127. doi: 10.1097/ DER.0000000000000263. PMID: 28169852

35. Noriega Salmón V. El propóleos, otro recurso terapéutico en la práctica clínica. 2014. Disponible en: https://repositorio.unican.es/xmlui/bitstream/handle/10902/5580/NoriegaSalmonV.pdf

36. Deswal H, Singh Y, Grover HS, Bhardwaj A. Healing effect of propolis in medicine and dentistry: a review. Innov J Ayruvedic Sci. 2016; 4(1):1-4. Disponible en: https:// api.semanticscholar.org/CorpusID:55393747

37. Parolia A, Thomas M, Kundabala M, Mohan M. Propolis and its potential uses in oral health. International JMMS. 2010;2(7):210-215. Disponible en: http://eprints. manipal.edu/id/eprint/1932

38. Downie W. Studies with pain rating scales. Ann Rheum Dis. 1979; 37(4): 378-81. doi: 10.1136 / ard.37.4.378

39. Macias M. Acción del propóleos sobre el tiempo de cicatrización en heridas postquirúrgicas. 2018. Tesis de Maestría. Universidad Nacional de La Plata. Disponible en: http://sedici.unlp.edu.ar/bitstream/handle/10915/66129/Documento_completo.pdf-PDFA. pdf?sequence $=1$ \&isAllowed $=y$

40. Basavaiah N, Suryakanth D. Propolis and allergic reactions. J Pharm Bioallied Sci. 2012;4(4):345. doi: 10.4103/0975-7406.103279

41. Budimir V, Brailo V, Alajbeg I, Vučićević Boras V, Budimir J. Allergic contact cheilitis and perioral dermatitis caused by propolis: case report. Acta Dermatovenerol Croat. 2012;20(3):187-90. 